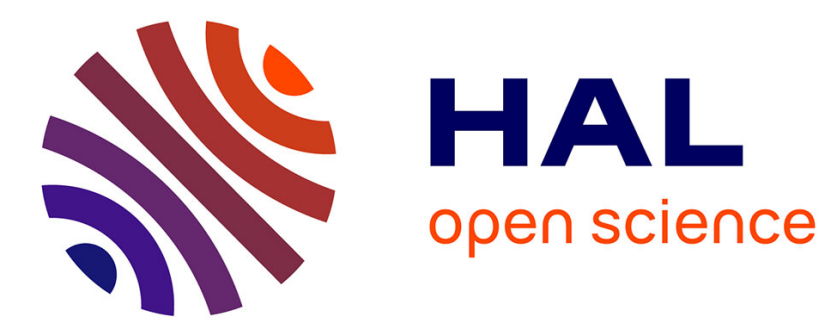

\title{
Sticky elastic collisions
}

J. Bec, Stefano Musacchio, Sankar Ray Samriddhi

\section{- To cite this version:}

J. Bec, Stefano Musacchio, Sankar Ray Samriddhi. Sticky elastic collisions. Physical Review E: Statistical, Nonlinear, and Soft Matter Physics, 2013, 87, pp.063013. hal-00874603

\section{HAL Id: hal-00874603 https://hal.science/hal-00874603}

Submitted on 18 Oct 2013

HAL is a multi-disciplinary open access archive for the deposit and dissemination of scientific research documents, whether they are published or not. The documents may come from teaching and research institutions in France or abroad, or from public or private research centers.
L'archive ouverte pluridisciplinaire HAL, est destinée au dépôt et à la diffusion de documents scientifiques de niveau recherche, publiés ou non, émanant des établissements d'enseignement et de recherche français ou étrangers, des laboratoires publics ou privés. 


\title{
Sticky elastic collisions
}

\author{
Jérémie Bec, ${ }^{1}$ Stefano Musacchio, ${ }^{2}$ and Samriddhi Sankar Ray ${ }^{3}$ \\ ${ }^{1}$ Laboratoire Lagrange, UMR 7293, Université de Nice Sophia Antipolis, CNRS, Observatoire de la Côte d'Azur, \\ Bd. de l'Observatoire, 06300 Nice, France \\ ${ }^{2}$ CNRS, Laboratoire J.A. Dieudonné UMR 7351, Université de Nice Sophia Antipolis, Parc Valrose, 06108 Nice, France \\ ${ }^{3}$ International Centre for Theoretical Sciences, Tata Institute of Fundamental Research, Bangalore 560012, India
}

(Received 19 March 2013; published 19 June 2013)

\begin{abstract}
The effects of purely elastic collisions on the dynamics of heavy inertial particles are investigated in a threedimensional random incompressible flow. It is shown that the statistical properties of interparticle separations and relative velocities are strongly influenced by the occurrence of sticky elastic collisions - particle pairs undergo a large number of collisions against each other during a small time interval over which, hence, they remain close to each other. A theoretical framework is provided for describing and quantifying this phenomenon and it is substantiated by numerical simulations. Furthermore, the impact of hydrodynamic interactions is discussed for such a system of colliding particles.
\end{abstract}

DOI: 10.1103/PhysRevE.87.063013 PACS number(s): 47.53.+n, 05.45.Df, 47.57.Gc, 47.27.eb

\section{INTRODUCTION}

Dust, droplets, bubbles, and other finite-size particles suspended in turbulent flows are common in nature [1-3]. Their statistical properties are very different from those of tracers, i.e., pointlike particles with the same mass density as the advecting fluid. Indeed, when the suspended particles have a finite size and a density different from that of the fluid, inertial effects become important. Consequently, the motion of particles starts differing from the underlying flow. This results in complex correlations between the positions of particles and the geometry of the turbulent flow: Heavier particles are expelled from vortical structures while lighter particles concentrate in their cores. A consequence of this is the presence of strong fluctuations in the spatial distribution of particles. This phenomenon, known as preferential concentration, has been the subject of extensive research in fluid dynamics for some decades [4,5].

A second consequence of inertia is that particles are likely to be very close to each other with velocity differences between them that are much larger than the fluid velocity differences evaluated at the particle positions. The process leading to such events, known as either the sling effect [6] or the formation of caustics [7], has been extensively measured and studied during the past ten years $[8,9]$. The presence of such spatial inhomogeneities is known to strongly alter possible interactions between particles. Since the pioneering work of [10], motivated by coalescences of cloud droplets, much work has been devoted to understanding the rate at which heavy inertial particles collide. A commonly adopted approach consists in assuming that the intercollision time is much longer than the time scale for the particle dynamics to converge to a statistically stationary regime. This premise, which is asymptotically true in the limit of very dilute suspensions, allows the counting of collisions without having to effectively perform them. The frequency at which such ghost particles collide is then a time-independent, statistical observable that can be quantified as a function of the size of the particles and their response time. Several studies have assumed this ghost-particle hypothesis in order to estimate collision rates of heavy inertial particles in turbulent flows [6,11]. However, little is known about the limits of such an approach. For instance, an important statistical weight is given to events when two concentrated clouds of particles cross each other with a large velocity difference. It is clear that the very dilute approximation should then fail and that multiple collisions are likely to occur. In this paper, by combining analytical methods and results from numerical simulations, we uncover an interesting effect of actual collisions on the dynamics and statistics of inertial particles transported by a nonstationary fluid flow.

The simplest framework for treating short-range collective effects in the particle dynamics is to consider an ensemble of hard spheres that are suspended in a prescribed flow and which undergo purely elastic (momentum- and energy-preserving) collisions with each other. Such a system, in the absence of any underlying fluid transport, has long been a paradigmatic model in statistical mechanics. It is the basis, for example, of the kinetic theory of gases, in which it is assumed that the kinetic energy is conserved both by the collisions and by the dynamics of each particle. If, however, we allow for a certain amount of inelasticity in the collisions a suitable model for granular gases is obtained [12]. A peculiar behavior of such granular systems is the spontaneous aggregation of particles. Since inelasticity implies the loss of a finite percentage of kinetic energy at each collision, the particles can eventually stick together, leading to the formation of large clusters. However, this effect occurs also in settings that are not common in studies of granular media. Indeed, as we will see in this work, it suffices that kinetic energy dissipation occurs not via collisions but rather through individual particle dynamics. This is the case for heavy inertial particles whose motion is dominated by viscous damping even when they undergo purely elastic collisions. We show that the clustering phenomenon emerging in such systems originates from what we call sticky elastic collisions. During these events, the particles bounce many times against each other and energy is dissipated during their motion between successive collisions. This mechanism has strong influences on the statistics of interparticle distances and relative velocities. Furthermore, to validate the presence of this effect in a more realistic setting, we study the influence 

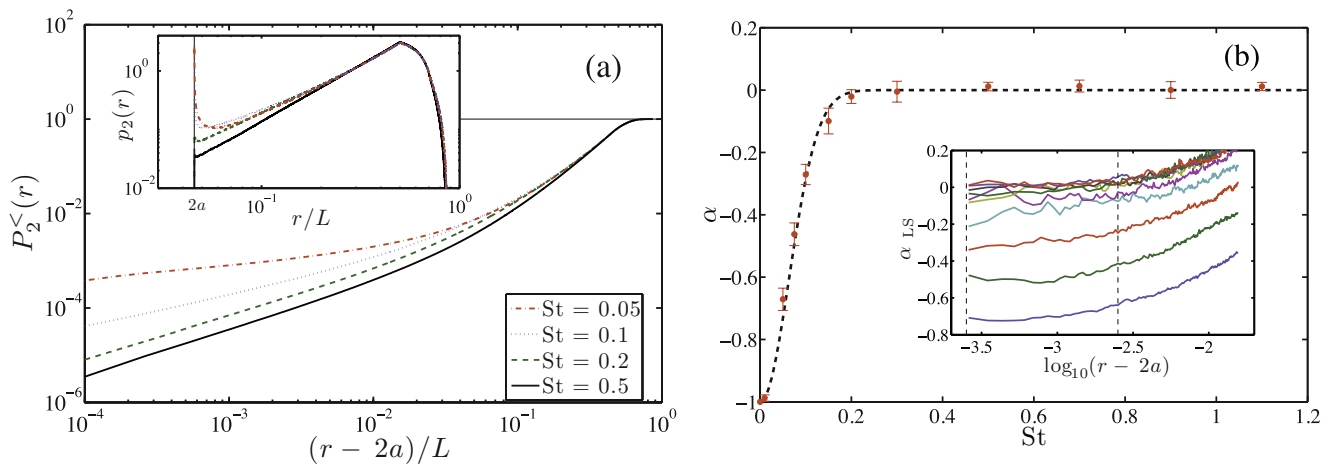

FIG. 1. (Color online) (a) Log-log plot of the cumulative probability distribution $P_{2}^{<}(r)$ as a function of $(r-2 a)$ for some representative values of St as indicated in the legend. Inset: Log-log plot of the two-particle density $p_{2}(r)=d P_{2}^{<}(r) / d r$ as a function of $r$ for the same values of St. (b) Exponent $\alpha$ versus St. The gray dashed line is an empirical fit of the form $-\exp \left[-(\mathrm{St} / 0.09)^{2}\right]$. Inset: local slopes $\alpha_{\mathrm{LS}}=$ $d \ln P_{2}^{<}(r-2 a) / d \ln (r-2 a)-1$ as a function of $(r-2 a)$ for various St. The two dashed vertical lines indicate the region over which we calculate the mean and the standard deviation of $\alpha_{\text {LS }}$.

of a second, long-range interaction, namely, the hydrodynamic interactions, and show that they cannot prevent sticky elastic collisions from occurring. We note in passing that there has been extensive work in recent years on the effect of both hydrodynamic interactions and going beyond the point-particle approach; however, such studies do not concentrate on the small-scale distribution of particles when hydrodynamic and interparticle interactions are included. In this work we focus on the small-scale distributions and see the effect of collisions and hydrodynamic interactions on them.

This paper is organized as follows. In Sec. II, we discuss the equations of motion of the inertial particles and recall some key results, in the absence of collisions, on preferential concentration. In Sec. III, we present numerical results and provide an asymptotic analysis for the phenomenon of sticky elastic collisions in the absence of hydrodynamic interactions. We then discuss the effect of hydrodynamic interactions in Sec. IV by considering only the far-field interactions. We make some concluding remarks and summarize our results, as well as provide a perspective for future work, in Sec. V.

\section{THE MODEL}

To set the stage, we begin by recalling the basic physics of a system of $N$ small hard spheres which are in a random, time-dependent, incompressible fluid field $\mathbf{u}(\mathbf{x}, t)$ and are subject to viscous dissipation. When such particles have a small Reynolds number and are much heavier than the fluid, they interact with the flow by Stokes viscous drag and their trajectories $\mathbf{x}_{i}(t)$ are determined by Newton's law:

$$
\dot{\mathbf{x}}_{i}=\mathbf{v}_{i}, \quad \dot{\mathbf{v}}_{i}=-\frac{1}{\tau}\left[\mathbf{v}_{i}-\mathbf{u}\left(\mathbf{x}_{i}, t\right)\right], \quad i \in[1, N] .
$$

Here $\tau$ is the viscous-drag relaxation (Stokes) time defined via $\tau=2 \rho_{\mathrm{p}} a^{2} /\left(9 \rho_{\mathrm{f}} v\right)$, where $\rho_{\mathrm{p}}$ is the particle density, $\rho_{\mathrm{f}}$ the fluid density, $v$ its kinematic viscosity, and $a$ the particle radius. The Stokes number is a measure of the inertia of the particle and is defined as $\mathrm{St}=\tau / \tau_{\mathrm{f}}$, where $\tau_{\mathrm{f}}$ is a characteristic time scale of the fluid flow. In this work, we additionally introduce interactions between particles via elastic collisions. This, for the case of spherical particles of equal size and mass, corresponds to an exchange of the radial components of the velocities of the two colliding particles upon impact, namely, when $\left|\mathbf{x}_{i}-\mathbf{x}_{j}\right|=2 a$.

The phenomenon of clustering occurs naturally, independent of the carrier flow compressibility, for an ensemble of particles which evolve according to Eq. (1), even in the absence of interactions or collisions between particles. We note that the case of no collisions is equivalent to the ghost-particle approach. The physical mechanisms which lead to strong inhomogeneities in the spatial distribution of particles arise from the underlying dissipative chaotic dynamics: The system is characterized by a constant contraction rate $d / \tau$ in the position-velocity phase space which drives the particles towards a dynamically evolving fractal set. The clusters of particles are the projections of such fractals on the position space. The fractal dimension of a distribution of particles provides a convenient tool to quantify their clustering [13-15]. In particular the correlation dimension $D_{2}$ is defined via the power-law behavior of the probability distribution function (PDF) of interparticle distances $p_{2}(r) \sim r^{D_{2}-1}$ [see the inset of Fig. 1(a) for particles with different Stokes numbers in a three-dimensional random flow]. Note that the density $p_{2}(r)$ is such that the probability that two particles are at a distance between $r$ and $r+d r$ is $p_{2}(r) d r$ and relates to the radial distribution function $g(r)$ : in three dimensions, one has $p_{2}(r)=4 \pi r^{2} g(r)$.

The intensity of clustering is influenced by the properties of the velocity field $\mathbf{u}(\mathbf{x}, t)$ and, in particular, by its spatial and temporal correlations. In this paper we will assume that (i) the velocity field is incompressible $\nabla \cdot \mathbf{u}=0$, (ii) it is differentiable in space and time, and (iii) it is characterized by unique time and length scales. Let us denote by $L_{\mathrm{f}}$ the fluid flow correlation length, which is taken to be much larger than the particle radius $a$, and by $\tau_{\mathrm{f}}$ its correlation time, which is of the same order as the turnover time $L_{\mathrm{f}} / U ; U$ is the typical amplitude of the velocity vector $\mathbf{u}$. In the limit of vanishing particle response time $\mathrm{St}=\tau / \tau_{\mathrm{f}} \rightarrow 0$, Eq. (1) becomes that of tracers, namely, $\dot{\mathbf{x}}_{i}=\mathbf{u}\left(\mathbf{x}_{i}, t\right)$, and the incompressibility condition ensures a uniform distribution of particles. Particles are distributed uniformly also in the opposite limit $\mathrm{St} \rightarrow \infty$, in which the force acting on particles become very small so 
that they follow almost a ballistic motion and fill the whole position-velocity phase space. The maximum of clustering is achieved for intermediate (order-unity) values of St where the minimum of the fractal dimension is reached.

How far does this picture change when we introduce interparticle interactions such as collisions? Clearly, collisions will affect the distribution of particles at scales of the order of their size. Heuristically, one expects the two-particle distribution $p_{2}(r)$ to be unchanged at separations $r$ much larger than the interaction-scale $2 a$. At separations of the order of $2 a$, the elastic collisions decorrelate the particle dynamics from the carrier flow. Naively, one would then expect that the particles are distributed like an ideal gas at such scales, and consequently have a uniform distribution. In dilute systems, the crossover between these two regimes would occur at a scale given by the distance traveled by the particles before their dynamics relaxes to its attractor. This distance can be written as $r_{\star}=\tau v_{\mathrm{c}}(\mathrm{St})$, where $v_{c}$ is the typical velocity difference at collisions. However, as seen from the inset of Fig. 1(a), this naive picture is wrong. As we will see in the next section, we indeed find that the two-particle density $p_{2}$ diverges when $r \rightarrow 2 a$. This effect is due to the presence of sticky elastic collisions.

\section{PARTICLE ADHESION THROUGH RECURRENT COLLISIONS}

To investigate the effect of collisions on clustering we resort to numerical simulations of Eq. (1). For simplicity and without any loss of generality, we consider two $(N=2)$ particles in a three-dimensional cubic domain of size $L$ with periodic boundary conditions. The velocity field is obtained from a superposition of Fourier modes whose amplitudes are stochastic Ornstein-Uhlenbeck processes with Gaussian statistics and a correlation time $\tau_{\mathrm{f}}$. The amplitude of each mode, which has a standard deviation of the order of $L / \tau_{\mathrm{f}}$, is chosen to ensure statistical isotropy at small scales. Such a random flow can be seen as a solution to the stochastically forced Stokes equation and mimics the behavior of the dissipative scales of a turbulent flow. For more details on the description of the random flow, please refer to [16]. In our simulations we use several values of $\tau$ with Stokes numbers $\mathrm{St}=\tau / \tau_{\mathrm{f}}$ lying between 0.01 and 1.1. We have done simulations with various values of the particle radius $a$, measured with respect to the box size $L=1.0$, but report here results obtained for $a \approx 0.02$ and a volume fraction $\phi=10^{-4}$. We have performed simulations for other radii ranging from $a \approx 0.005\left(\phi=10^{-6}\right)$ to $a \approx 0.1\left(\phi=10^{-2}\right)$ and found that our results are consistent with the ones being reported in this paper. Our time marching is an implicit Euler scheme with a fixed time step $\delta t=10^{-4}$ when the particles are far away from each other. However, when the particles are close to each other, the time step is adapted in order to resolve collisions with a high accuracy.

We have also performed simulations in two dimensions (2D) and with a larger number of particles $(N=1024$ and 2048) and volume fractions in the range $10^{-6} \leqslant \phi \leqslant 10^{-3}$. The results reported in this paper do not change with either the dimension (as is also predicted by our asymptotic analysis as shown below) or the number of particles $N$ and the volume fractions, as long as they are sufficiently dilute. Thus, in this paper, we present results only for the $3 \mathrm{D}$ case and for two particles. This simplification helps us to elucidate the central idea of our work, as well as making the calculations for the hydrodynamic interaction easier (see Sec. IV).

Our numerical simulations reveal a very interesting phenomenon which is clearly absent in the collisionless or ghostcollision case. As expected the effects of collisions on the spatial distribution of particles, as characterized by the PDF of the interparticle distance, are negligible for particle separations much larger than $2 a$. However, collisions dramatically affect the statistics of pair separations at small scales. In particular, the probability distribution function of the interparticle distance $p_{2}(r)$ displays a power-law behavior $p_{2}(r) \sim(r-2 a)^{\alpha}$ for distances close to the cutoff $a$. The exponent $\alpha$ is a monotonically increasing function of the Stokes number St: It begins with the value -1 for $\mathrm{St} \rightarrow 0$ and approaches 0 as $\mathrm{St} \rightarrow \infty$. Figure 1(a) shows the cumulative probability distribution $P_{2}^{<}(r)$, which is the probability of finding two particles at a distance less than $r$, as a function of $(r-2 a)$, on a log-log scale, for some representative values of St that we use in our simulations. We clearly find that $P_{2}^{<}$behaves as a power law proportional to $(r-2 a)^{\alpha+1}$ at small values of $r-2 a$. From a local slope analysis we extract the local scaling exponent $\alpha_{\mathrm{LS}}=d \ln P_{2}^{<}(r-2 a) / d \ln (r-2 a)-1$ [see the inset of Fig. 1(b)]; the mean of this gives us a measure of the scaling exponent $\alpha$ and the standard deviation an estimate of the error. In Fig. 1(b) we show the behavior of $\alpha$ as a function of the Stokes number. The gray dashed line is an empirical fit to our numerical data and is given by $-\exp \left[-(\mathrm{St} / 0.09)^{2}\right]$. In the inset the dashed vertical lines denote the region over which we calculate the mean and the standard deviation to obtain $\alpha$. Two asymptotic values are clearly visible on Fig. 1(b): one observes that $\alpha \rightarrow 0$ when St $\rightarrow \infty$ and $\alpha \rightarrow-1$ when $\mathrm{St} \rightarrow 0$. Note that the stiffness of the system in the limit St $\rightarrow 0$ prevents us from obtaining accurate numerical results in this limit. Nevertheless, our data show a monotonic convergence towards $\alpha=-1$. These two asymptotic values of the exponent are signatures of two different collision mechanisms which we explain below.

For large Stokes numbers the motion of particles is weakly correlated with the local value of the fluid velocity field. This is because the particle motion is determined by the cumulative contribution of the flow integrated over the particle trajectories with a long memory kernel. When two particles collide, the velocities of the two particles are almost uncorrelated and vary on very large time scales. The relative motion of these two particles is therefore almost ballistic and with a random relative velocity. The time that the two particles spend at a distance between $r$ and $r+d r$ is given by $d t=V d r$, where $V$ is their typical velocity difference. This leads to $p_{2}(r) \sim(r-2 a)^{0}$, thus yielding $\alpha \rightarrow 0$ when $\mathrm{St} \rightarrow \infty$.

The limit of $\mathrm{St} \rightarrow 0$ is more complicated. In this case the motion of the particles is strongly correlated with the fluid velocity field $\mathbf{u}$ and, therefore, the particles typically collide with a very small relative velocity. This relative velocity is of the order of the fluid velocity difference, namely, $2 a \sigma$, where $\sigma$ is the local gradient of $\mathbf{u}$. After the collision they tend to separate but the fluid velocity field quickly brings them back together, because of the short relaxation time $\tau \ll \tau_{\mathrm{f}}$. 

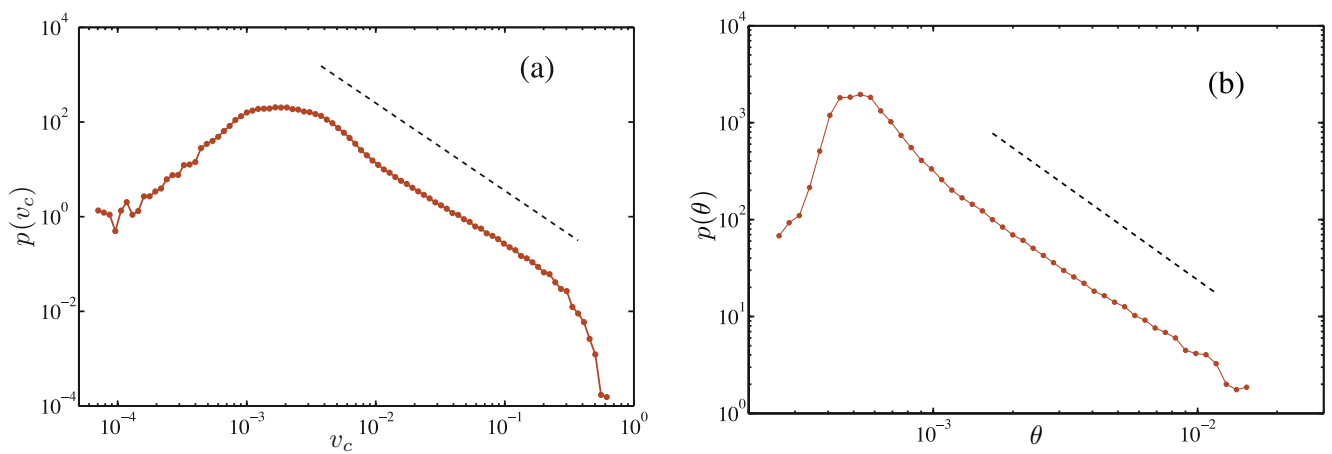

FIG. 2. (Color online) (a) Log-log plot of the PDF of the intercollision radial velocity $v_{\mathrm{c}}$ for $\mathrm{St}=0.01$; the dashed line shows a scaling of -1.85. (b) Log-log plot of the PDF of the intercollision time $\theta$ for $\mathrm{St}=0.01$; the dashed line shows a scaling of -1.95 .

The particles then collide again and this mechanism will, therefore, lead to a long series of high-frequency collisions during which the particles remain within a distance of the order of $2 a$. Next, to quantify the effects of these events on the statistics of interparticle distance, let us consider a simple, one-dimensional model for the separation $r=\left|\mathbf{x}_{1}-\mathbf{x}_{2}\right|$ and the radial relative velocity $v=\left(\mathbf{v}_{1}-\mathbf{v}_{2}\right) \cdot \hat{\boldsymbol{r}}$ of two particles, between two collisions occurring at times $t_{n}$ and $t_{n+1}$. The equations of motion can be written as

$$
\dot{r}=v, \quad \tau \dot{v}=-v-2 a \sigma .
$$

Here, we have assumed that the fluid velocity gradient $-\sigma<0$ remains constant. This is justified because the particle dynamics is much faster than the correlation time of the fluid velocity $\left(\tau \ll \tau_{\mathrm{f}}\right)$. Also, we have neglected higher-order terms in the Taylor expansion of $\mathbf{u}$. The above equation gives $r(t)=2 a-\tau\left(v_{n}+2 a \sigma\right)[\exp (-t / \tau)-1]-2 a \sigma t$ and $v(t)=v_{n} \exp (-t / \tau)+2 a \sigma[\exp (-t / \tau)-1]$, where $v_{n}$ is the relative radial velocity immediately after the $n$th impact at time $t_{n}$. By introducing the small parameter $\epsilon_{n}=v_{n} /(2 a \sigma)$ and Taylor-expanding the above expressions for $t / \tau \ll 1$, one obtains a recursive relation for the relative velocity at collision, namely, $\epsilon_{n+1}=\epsilon_{n}\left(1-2 \epsilon_{n} / 3\right)$, which leads to $v_{n} \sim 2 a \sigma / n$. The intercollision time $\theta_{n}=t_{n+1}-t_{n}$ decreases as $\theta_{n} \sim \tau / n$, and the maximum separation distance $r^{*}$ reached by the two particles in the excursions between two collisions scales as $\delta=r_{n}^{*} /(2 a)-1 \sim \sigma \tau / n^{2}$. The number of collisions increases exponentially in time $n_{c} \sim \exp (C t / \tau)$, with $C>0$, and the interparticle distance goes to 0 . We call this phenomenon sticky elastic collisions. Note that the series defined by the sum of intercollision times $\sum_{n} \theta_{n}$ does not converge. This ensures that the number of collisions does not become infinite in a finite time, at variance with the case for inelastic collisions where the particles eventually collapse and aggregate. In the case of the one-dimensional sticky elastic collisions, the recurrent process stops only when the fluid velocity gradient becomes positive (for $t \simeq \tau_{\mathrm{f}}$ ) and takes the two particles away from one another. During any one of these events, only the first $m$ collisions will contribute to the probability of having the two particles at a distance larger than $r \simeq 2 a\left(1+\sigma \tau / m^{2}\right)$. The fraction of time spent at a distance larger than $r$ is thus $\sim \sum_{n<m} \theta_{n} \sim \ln m \sim-\ln (r-2 a)$. This gives for the PDF of the interparticle distances $p_{2}(r) \sim(r-2 a)^{-1}$, yielding the asymptotic value $\alpha \rightarrow-1$ for $\mathrm{St} \rightarrow 0$.
The heuristic arguments developed here to quantify the statistical signature of sticky events are purely one dimensional. However, they extend to higher dimensions only via geometrical considerations. The one-dimensional case would hold true if the velocity difference $\mathbf{v}$ between the particles were exactly aligned with their separation $\mathbf{r}$. A misalignment leads to rebounds of the two particles at different locations on their surfaces. For spherical particles, this implies that the better is the alignment between $\mathbf{v}$ and $\mathbf{r}$, the higher is the number of successive secondary collisions. Statistically, this implies that the distribution of distances is dominated by almost head-on collisions, which can essentially be treated as a one-dimensional problem. The space dimensionality should just appear as a multiplicative factor in the power-law behavior at $r \rightarrow 2 a$. We have indeed performed simulations in 2D (and with a large number of particles), as stated before, and have found the same signatures of sticky elastic collisions as has been seen in the three-dimensional, two-particle, simulations [Fig. 1(b)].

The effects of these events are also detectable in the statistics of the intercollision times $\theta$ and the relative radial velocity at collision $v_{\mathrm{c}}$. Within the model derived above, for $\mathrm{St} \rightarrow 0$, we obtain $p\left(v_{\mathrm{c}}\right) \sim v_{\mathrm{c}}^{-2}$ and $p(\theta) \sim \theta^{-2}$. In Figs. 2(a) and 2(b) we show $\log$ - $\log$ plots of $p\left(v_{\mathrm{c}}\right)$ versus $v_{\mathrm{c}}$ and $p(\theta)$ versus $\theta$, respectively, obtained from simulations for $\mathrm{St}=0.01$; the dashed lines show a scaling exponent of $\approx-1.9$ in each case. We have checked that the scaling becomes significantly shallower and the extent of the scaling gets progressively reduced when $\mathrm{St}$ increases. Note that a similarly steep increase of the distribution of intercollision times at small values has been observed in [17] in full direct numerical simulations of finite-size particles suspended in a viscous flow. This effect has been interpreted as a possible consequence of lubrication forces between the particles that makes them remain close to each other for long times. In the next section, we will comment on the influence of hydrodynamical interactions on sticky elastic collisions. Nevertheless, it is worth stressing here that lubrication is not necessary to obtain multiple collisions between particles.

The tails of $p\left(v_{\mathrm{c}}\right)$ and $p(\theta)$ at small values seem to indicate that they cannot be normalized. However, we observe for small but finite values of St, a cutoff at the smallest values of $v_{\mathrm{c}}$ and $\theta$, which prevents this divergence. Indeed, the power-law behaviors are due to typical sticky events. As we have seen, 
the number of collisions is of the order of $n_{\mathrm{c}} \sim \exp (C / \mathrm{St})$. Therefore, the minimal collisional velocity and intercollision time are both proportional to $1 / n_{c} \sim \exp (-C / \mathrm{St})$. The power law is thus just appearing in intermediate ranges, namely, $\left(2 a / \tau_{\mathrm{f}}\right) \exp (-C / \mathrm{St}) \ll v_{\mathrm{c}} \ll\left(2 a / \tau_{\mathrm{f}}\right)$ and $\tau \exp (-C / \mathrm{St}) \ll$ $\theta \ll \tau$. The events leading to values smaller than the lower bounds are related to situations where the fluid velocity gradient is maintained negative for an exceptionally long time. This happens with an inverse-Gaussian probability in a finite-correlation-time flow, whence the cutoff. Also, the extension of this argument to the singular limit $\mathrm{St}=0$ is far from obvious. Note, finally, that in dimensions higher than 1, the geometrical considerations explained above should also provide a cutoff for very small intercollision times and velocities.

\section{EFFECT OF HYDRODYNAMIC INTERACTIONS}

In this section, we address the question of whether or not sticky elastic collisions can be observed in more realistic flows. For that, it is important to know how far the observations and conclusions drawn above are valid when, in addition to collisions, we introduce hydrodynamic interactions between particles. To answer this question, we take into account the far-field or long-range interactions and assume that it is valid all the way to the smallest separations. This approach yields several interesting results which we discuss below. Of course, this assumption, in reality, breaks down when particles approach very close to one other. However, in that case, either lubrication leads to an effective increase in the particle physical radius or, in the case when the velocity difference is too large, hydrodynamics might not be a valid description of the interactions between the particles.

In its simplest formulation, the long-range hydrodynamic interactions between particles in a flow are taken into account by considering the perturbation in the ambient fluid velocity field, as experienced by an individual particle, because of the motion of all the other particles. Thus the effective velocity field acting on any particle is a superposition of the unperturbed (turbulent) advecting flow $\mathbf{u}(\mathbf{x}, t)$ and of the perturbation to this flow due to the other particles. In a system of $N$ particles, the net perturbation $\mathbf{u}^{(i)}$ on the flow field experienced by the $i$ th particle is obtained by summing over the contributions made by each of the other $(N-1)$ particles [18]:

$$
\mathbf{u}^{(i)}=\sum_{j \neq i} \mathbf{U}_{s}\left[\mathbf{r}_{i j}, \mathbf{v}_{j}-\mathbf{u}\left(\mathbf{x}_{j}, t\right)-\mathbf{u}^{(j)}\right],
$$

where $\mathbf{u}\left(\mathbf{x}_{j}, t\right)$ is the unperturbed fluid velocity at particle position $\mathbf{x}_{j}$, the separation between the $i$ th and $j$ th particles is given by $\left|\mathbf{r}^{(i j)}\right|$, and $\mathbf{U}_{s}$ is the Stokes flow around a sphere, given by

$\mathbf{U}_{s}(\mathbf{r}, \mathbf{v})=\left[\frac{3}{4} \frac{a}{r}-\frac{3}{4}\left(\frac{a}{r}\right)^{3}\right] \frac{\mathbf{r}}{r^{2}}(\mathbf{v} \cdot \mathbf{r})+\left[\frac{3}{4} \frac{a}{r}+\frac{1}{4}\left(\frac{a}{r}\right)^{3}\right] \mathbf{v}$.

Given the structure of the equations, for a system of $N$ particles, it is computationally challenging to solve exactly the perturbation field as it requires the solution of a linear $3 \mathrm{~N}$-dimensional system. Thus various approximations and iterative schemes become essential. However, in the present problem being studied in this paper, which involves only two particles, it is possible to solve exactly the hydrodynamic interaction term, as given in Eq. (3), because it involves merely the inversion of a $6 \times 6$ matrix.

Let us now understand the effect of hydrodynamic interactions on the statistics of particle collisions, from a theoretical point of view, by considering the following simplified model. We consider the case of two particles which approach each other with velocities $\mathbf{v}_{1}=-\mathbf{v}_{2}$ aligned along their separation vector $\mathbf{r}$. Under the assumptions already described in Sec. III, the unperturbed fluid velocity field at the position of the $i$ th particle $\mathbf{u}\left(\mathbf{x}_{j}, t\right)$ is aligned with the separation vector $\mathbf{r}$ and the unperturbed fluid velocity field vanishes at the midpoint of of the separation of the particles. A direct consequence of this is that the perturbation $\mathbf{u}^{(1)}$ on particle 1 due to particle 2 is equal and opposite to the perturbation $\mathbf{u}^{(2)}$ on particle 2 due to particle 1, i.e., $\mathbf{u}^{(1)}=-\mathbf{u}^{(2)}$.

Under the above assumptions, the perturbation field experienced by particle 2, obtained from Eqs. (3) and (4), reduces to

$$
\mathbf{u}^{(2)}=\left[\mathbf{v}_{1}-\mathbf{u}\left(\mathbf{x}_{1}, t\right)-\mathbf{u}^{(1)}\right]\left(\frac{3}{2} \frac{a}{|\mathbf{r}|}-\frac{1}{2} \frac{a^{3}}{|\mathbf{r}|^{3}}\right) .
$$

Since, at the point of collision, $|\mathbf{r}|=2 a$, and by using $\mathbf{u}^{(1)}=$ $-\mathbf{u}^{(2)}$, one has

$$
\mathbf{u}^{(1)}=-\frac{11}{5}\left[\mathbf{v}_{1}-\mathbf{u}\left(\mathbf{x}_{1}, t\right)\right] .
$$

Finally we substitute the above estimate in the equation of motion for particle 1 ,

$$
\frac{d \mathbf{v}_{1}}{d t}=-\frac{1}{\tau}\left[\mathbf{v}_{1}-\mathbf{u}\left(\mathbf{x}_{1}, t\right)-\mathbf{u}^{(1)}\right],
$$

to eventually obtain

$$
\frac{d \mathbf{v}_{1}}{d t}=-\frac{16}{5 \tau}\left[\mathbf{v}_{1}-\mathbf{u}\left(\mathbf{x}_{1}, t\right)\right] .
$$

The above analysis shows that the effect of long-range hydrodynamic interactions reduces, in the vicinity of collisions, to the dynamics of a system without such interactions but with an effective Stokes number, which is equal to the actual Stokes number reduced by a factor of $16 / 5=3.2$. Thus a system of particles with a Stokes number St and subject to hydrodynamic interactions can be replaced by a system of particles, without any hydrodynamic interactions but with an effective Stokes number $\mathrm{St}_{\text {eff }}=(5 / 16)$ St when we consider their statistical properties for very small interparticle separations within the framework of our model. It should be stressed that this surprising result is obtained only for particles very close to each other and it certainly does not hold for individual trajectories of particles when they are well separated.

To confirm the arguments presented above we resort once more to numerical simulations by implementing the long-range hydrodynamic interactions (3). We use values of St between 0.05 and 1.1 as we had used for the case without any interaction terms. We begin by measuring the values of the exponent $\alpha$ introduced in the previous Section that describes the behavior of the inter-particle distance distribution. Figure 3(a) shows the values of $\alpha$ obtained as 

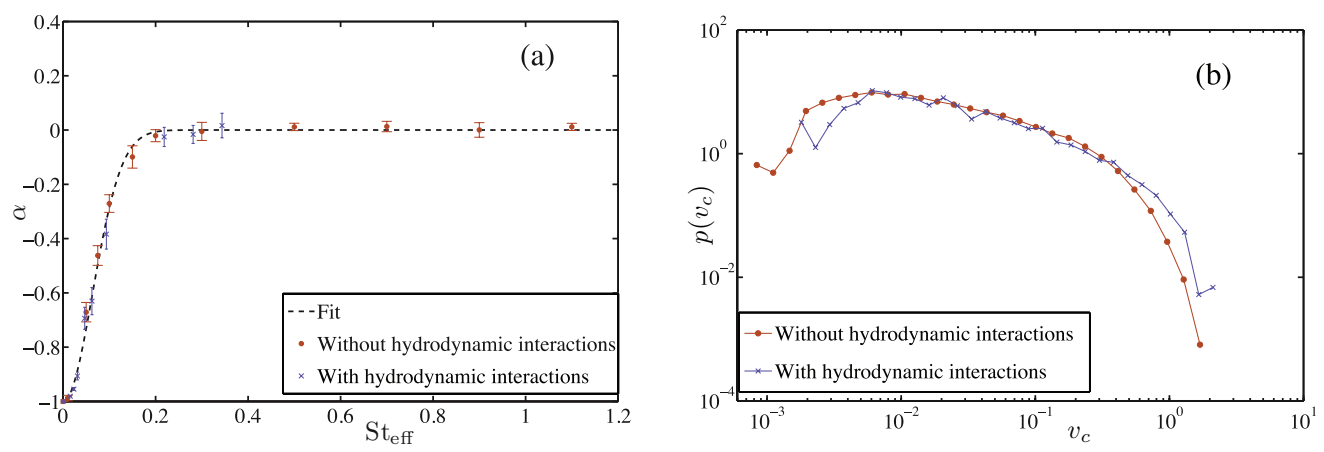

FIG. 3. (Color online) (a) Exponents $\alpha$ of the two-particle distribution $p_{2}(r) \propto(r-2 a)^{\alpha}$, along with their error bars. The case with hydrodynamic interactions $(x)$ is represented versus $\mathrm{St}_{\mathrm{eff}}=(5 / 16) \mathrm{St}$ and that without interactions $(\bullet)$, as a function of St; the gray dashed line is the empirical fit $-\exp \left[-(\mathrm{St} / 0.09)^{2}\right]$ discussed in the previous section. (b) PDF of the intercollision radial velocity $v_{\mathrm{c}}$ obtained from simulations with hydrodynamic interactions $(\times)$ for $\mathrm{St}=0.15$, that is, $\mathrm{St}_{\mathrm{eff}}=0.047$ and without hydrodynamic interactions $(\bullet)$ for $\mathrm{St}=0.05$.

a function of the effective Stokes number $\mathrm{St}_{\mathrm{eff}}=(5 / 16) \mathrm{St}$ (crosses); our data seems to fall, within error bars, on the empirical fit shown by a dashed gray line. To make the comparison more illuminating we plot on the same graph and as a function of the actual Stokes number St, the values of $\alpha$ (black dots) obtained from numerical simulations without hydrodynamic interactions, and already shown in Fig. 1(b). Furthermore, in Fig. 3(b) we show the PDF of the intercollision velocity $p\left(v_{\mathrm{c}}\right)$ as a function of $v_{\mathrm{c}}$, on a $\log$ - $\log$ scale, for $\mathrm{St}=0.05$ obtained from a simulation without hydrodynamic interactions (black dots) and for $\mathrm{St}=0.15$ obtained from a simulation with hydrodynamic interactions (crosses). The two PDFs are nearly overlapping, as our arguments before would suggest that the effective Stokes is $\mathrm{St}_{\text {eff }}=0.047 \approx 0.05$ for the case with the hydrodynamic interactions.

Our results suggest that hydrodynamic interactions increase the efficiency of dissipative mechanisms in terms of a reduction of the effective Stokes number. However, such considerations can lead to only qualitative deductions as our study accounts for long-range interactions only. The effect of lubrication forces will become dominant for particles at very small separations. On the one hand, this type of interaction is expected to decrease the collision efficiency between particles [19]. On the other hand, lubrication is expected to increase damping when particles get close to each other; this effect is usually modeled by a restitution coefficient less than unity. Because of these two competing mechanisms, it is difficult to predict whether short-range hydrodynamic interactions will enhance or diminish the sticky elastic collision phenomenon. A more detailed and realistic simulation which includes lubrication is beyond the scope of the present work.

\section{CONCLUSIONS}

In our work we have considered the effect of elastic collisions on the clustering of inertial particles in a very simplified model which allows precise analytical predictions and the testing of the same via accurate simulations. In particular we have investigated their influence on the probability distribution of interparticle distance. Surprisingly, our findings differ markedly from the naive picture that collisions might introduce only a small-scale molecular chaos. We observe that the small-distance statistics is dominated by a phenomenon which we call sticky elastic collisions, during which particles undergo a very large number of collisions during a time of the order of the fluid correlation time. It is interesting to note that these sticky elastic collisions remarkably resemble inelastic collapses observed in granular media, even though the underlying assumption in granular media (conservative intercollision dynamics and dissipative collisions) is exactly the opposite of what we have considered here. In addition we have investigated the effect on this phenomenon of long-range hydrodynamic interactions between particles. Our results seem to indicate that the most significant effect at small scales of such interactions is to introduce an effective Stokes number. The problem of investigating the effect on sticky elastic collisions of short-range hydrodynamical interactions requires more rigorous theoretical understanding and more elaborate numerical simulations which are left for future studies.

In this paper we have focused on two-particle interactions in three-dimensional random flows. It is clear that the

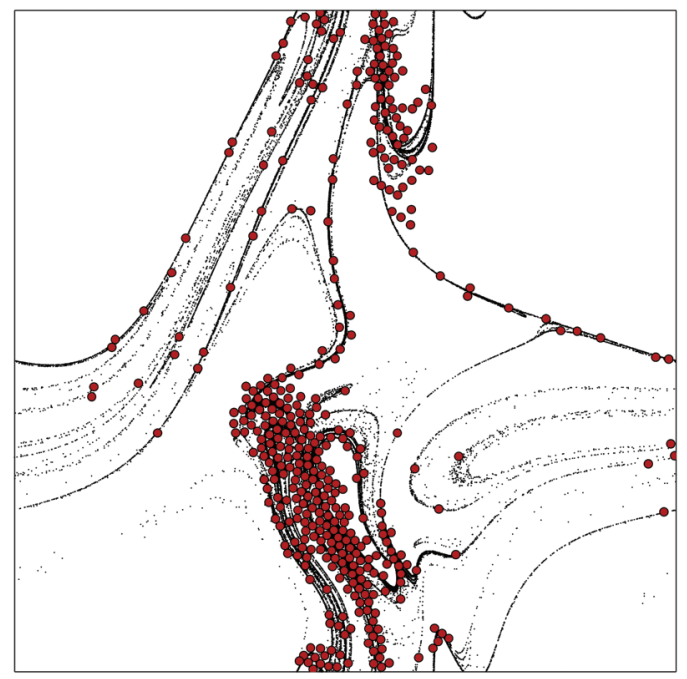

FIG. 4. (Color online) Instantaneous distribution of particles undergoing elastic collisions (red circles), together with the attractor of the dynamics (black dots). The results were here obtained in a two-dimensional periodic random flow for $N_{\mathrm{p}}=400$ particles, $\mathrm{St}=0.1$, and a surface fraction $\Phi=N_{\mathrm{p}} \pi a^{2} / L^{2}=0.05$. 
phenomenon of sticky elastic collisions is independent of the physical dimension and is present even for large numbers of interacting particles, as can be seen in our simulations of two-dimensional flows with a large number of particles. However, focusing on two-particle interactions is relevant only in extremely dilute settings. When the volume fraction of particles becomes large, the combination of particle clustering on a dynamically evolving fractal attractor and sticky elastic collisions can lead to interesting collective phenomena. This is illustrated in Fig. 4 for two dimensions, where one observes in the particle distribution the coexistence between packed and dilute phases depending on the local structure of the attractor.

Finally, let us turn back to the application of our work to the collisions between droplets suspended in a turbulent flow. There are still many open questions concerning the stability of coalescence processes for the high impact velocities that are observed in turbulent settings. In particular, estimates on relative velocities between meter-sized objects in circumstellar disks are by far too large to allow for their accretion and growth to form planet embryos [20,21]. The dissipative mechanisms relating to sticky elastic collisions are likely to play a role there. However, such issues must be investigated in a full direct numerical simulation which includes both short- and long-range hydrodynamical interaction terms.

\section{ACKNOWLEDGMENTS}

We would like to thank G. Falkovich and D. Mitra for useful discussions and acknowledge support from the European Cooperation in Science and Technology (EU COST) Action MP0806. The research leading to these results has received funding from the European Research Council under the European Community's Seventh Framework Program (FP7/2007-2013, Grant Agreement No. 240579) and from the Agence Nationale de la Recherche (Programme Blanc ANR-12-BS09-011-04).
[1] G. Csanady, Turbulent Diffusion in the Environment, Geophysics and Astrophysics Monographs Vol. 3 Reidel, Dordrecht, 1980).

[2] S. Post, and J. Abraham, Int. J. Multiphase Flow 28, 997 (2002).

[3] R. A. Shaw, Annu. Rev. Fluid Mech. 35, 183 (2003).

[4] S. Douady, Y. Couder, and M. E. Brachet, Phys. Rev. Lett. 67, 983 (1991).

[5] K. D. Squires and J. K. Eaton, Phys. Fluids A 3, 1169 (1991).

[6] G. Falkovich, A. Fouxon, and M. Stepanov, Nature (London) 419, 151 (2002).

[7] M. Wilkinson and B. Mehlig, Europhys. Lett. 71, 186 (2005).

[8] J. Bec, L. Biferale, M. Cencini, A. S. Lanotte, and F. Toschi, J. Fluid Mech. 646, 527 (2010).

[9] J. P. L. C. Salazar and L. R. Collins, J. Fluid Mech. 696, 45 (2012).

[10] P. G. Saffman and J. S. Turner, J. Fluid Mech. 1, 16 (1956).

[11] S. Sundaram and L. R. Collins, J. Fluid Mech. 335, 75 (1997).

[12] I. Goldhirsch, Annu. Rev. Fluid Mech. 35, 267 (2003).
[13] J. Bec, L. Biferale, M. Cencini, A. Lanotte, S. Musacchio, and F. Toschi, Phys. Rev. Lett. 98, 084502 (2007).

[14] E. Calzavarini, M. Kerscher, D. Lohse, and F. Toschi, J. Fluid Mech. 607, 13 (2008).

[15] M. Wilkinson, B. Mehlig, and K. Gustavsson, Europhys. Lett. 89, 50002 (2010).

[16] J. Bec, J. Fluid Mech. 528, 255 (2005).

[17] A. Ten Cate, J. J. Derksen, L. M. Portela, and H. E. A. Van Den Akker, J. Fluid Mech. 519, 233 (2004).

[18] L.-P. Wang, B. Rosa, H. Gao, G. He, and G. Jin, Int. J. Multiphase Flow 35, 854 (2009).

[19] L. P. Wang, O. Ayala, S. E. Kasprzak, and W. W. Grabowski, J. Atmos. Sci. 62, 2433 (2005).

[20] G. Wurm, J. Blum, and J. E. Colwell, Icarus 151, 318 (2001).

[21] M. Wilkinson, B. Mehlig, and V. Uski, Astrophys. J. Suppl. Ser. 176, 484 (2008). 